\title{
A Study into the Evolutionary Divergence of the Core Promoter Elements of PRPF31 and TFPT
}

Anna M Rose ${ }^{1 *}$, Amna Z Shah ${ }^{1}$, Giovanna Alfano1, Kinga M Bujakowska², Amy F Barker ${ }^{1}$, J Louis Robertson ${ }^{1}$, Sufia Rahman ${ }^{1}$, Lourdes Valdés Sánchez ${ }^{3}$, Francisco J Diaz-Corrales ${ }^{3}$, Christina F Chakarova ${ }^{1}$, Abhay Krishna ${ }^{3}$ and Shomi S Bhattacharya ${ }^{1}$

${ }^{1}$ Department of Genetics, UCL Institute of Ophthalmology, London, United Kingdom

${ }^{2}$ Ocular Genomics Institute \& Berman-Gund Laboratory, Department of Ophthalmology, Harvard Medical School, Boston, USA

${ }^{3}$ Andalusian Molecular Biology and Regenerative Medicine Centre (CABIMER), Seville, 41092, Spain

\begin{abstract}
Mutations in PRPF31 have been implicated in retinitis pigmentosa, a blinding disease caused by degeneration of rod photoreceptors. The disease mechanism in the majority of cases is haploinsufficiency. Crucially, attempts at generation of animal models of disease have proved unsuccessful, yielding animals with a visual phenotype that does not mirror human disease. This suggests that, in these animals, the transcriptional regulation of PRPF31 is different to humans and compared to other species. Study of the evolution of the PRPF31 core promoter has important implications for our understanding of human disease, as disease phenotype is modified by differentially expressed alleles in the population.
\end{abstract}

PRPF31 lies in a head-to-head arrangement with TFPT, a gene involved in cellular apoptosis. The two genes were shown to share common regulatory elements in the human genome. In this study, the core promoters of PRPF31 and TFPT were characterised by dual-luciferase reporter assay using genomic DNA from the green monkey, domestic dog and house mouse. It was found that the core promoters were conserved between human and monkey.

In dog, the TFPT core promoter was conserved, but different PRPF31 gene architecture meant the gene was controlled by a long-range promoter lying some $2000 \mathrm{bp}$ from the transcription start site.

There was very low level of conservation (<20\%) of the PRPF31 5 ' region between mouse and human. It was shown that mouse populations did not show variable Prpf31 expression levels, revealing a potential explanation for the lack of phenotype observed in the Prpf31 knock-out mouse model.

Keywords: Photoreceptor; Dual-luciferase reporter; PRPF31 mutation; TFPT

\section{Introduction}

PRPF31 encodes the ubiquitous splicing factor PRPF31, an essential component of the U4/U6.U5 tri-snRNP. The gene is highly conserved throughout evolution, with orthologues in all vertebrate species, invertebrates and lower species, including yeast. Mutations in PRPF31 have been shown to be a major cause of autosomal dominant retinitis pigmentosa (adRP), accounting for $5 \%$ of disease in the UK $[1,2]$.

A unique feature of PRPF31-associated adRP is phenotypic nonpenetrance, where within affected families there are asymptomatic mutation carriers. This is due to the existence of differentially expressed wildtype PRPF31 alleles, with co-inheritance of a PRPF31 mutation and a higher-expressing allele providing protection against clinical manifestation of disease. It has been shown that there is variable expression of PRPF31 in the general population and, that within mutation carrying families, asymptomatic mutation carriers have more than two-fold higher expression levels of wildtype PRPF31 compared to symptomatic individuals [3-6].

One study looked at phenotypic discordance between mutationcarrying siblings and observed that the symptomatic and asymptomatic siblings consistently inherited different wildtype chromosome $19 \mathrm{q} 13$ alleles from the non-mutation carrying parent [7]. It is generally thought, therefore, that cis-acting factors that affect the level of PRPF31 expression (such as regulatory region polymorphisms) underlie phenotypic non-penetrance in mutation-carrying families. However, attempts to identify such changes have not yet been successful.

It has also been demonstrated that there is increased expression of both PRPF31 alleles in asymptomatic mutation-carrying individuals, with subsequent degradation of the mutant molecule by nonsense mediated decay - this indicating that at least one factor that alters PRPF31 expression acts in trans [8]. One possible trans-acting factor was identified through the association of higher PRPF31 expression and an expression quantitative trait locus (eQTL) at 14q21-23, although the exact factor was not characterized [3]. It was also shown that variable expression of CNOT3 is an important factor in determining PRFP31 expression level - with increased levels of CNOT3 protein causing transcriptional repression of PRPF31 [9]. CNOT3 is a component of the Ccr4-Not transcription complex, which is a global regulator of RNA polymerase II-mediated transcription [10].

Attempts to generate mouse models of PRPF31-associated adRP have failed to yield animals with a retinal degeneration phenotype. Neither Prpf31 knock-in animals nor knock-out animals displayed retinal degeneration, and the animals did not have any visual defect at up to 18 months of age [11]. There is some evidence that Prpf31 knockout mice develop changes within the retinal pigment epithelium (RPE), with vacuolation, loss of the basal infoldings and accumulation

*Corresponding author: Anna M Rose, Department of Genetics, UCL Institute of Ophthalmology, 11-43 Bath Street, London, EC1V 9EL, UK, E-mail: anna.rose@ucl.ac.uk

Received June 26, 2013; Accepted August 20, 2013; Published August 22, 2013

Citation: Rose AM, Shah AZ, Alfano G, Bujakowska K, Barker AF, et al. (2013) A Study into the Evolutionary Divergence of the Core Promoter Elements of PRPF31 and TFPT. J Mol Genet Med 7: 67. doi:10.4172/1747-0862.1000067

Copyright: @ 2013 Rose AM, et al. This is an open-access article distributed under the terms of the Creative Commons Attribution License, which permits unrestricted use, distribution, and reproduction in any medium, provided the original author and source are credited 
of amorphous deposits between the RPE and Bruch's membrane [12]. There was not, however, death of retinal photoreceptor cells (the primary histological and pathological change in human disease) and no change in retinal function was reported - and so these animals cannot be considered a model for human disease [12]. It is possible that the same RPE changes are observed in asymptomatic individuals, but this study is not feasible. It can be concluded that in mouse, $50 \%$ of protein level is sufficient for normal retinal function.

Regulation of gene expression is central to pathogenesis of PRPF31 mutations in humans and also the failure of animal models of disease and it is necessary, therefore, to understand the 5' architecture of the PRPF31 gene. PRPF31 and TFPT are arranged in a bidirectional gene pair, with partially shared exon 1 , at chromosome $19 \mathrm{q} 13.4$. It is increasingly recognised that many genes exist in bidirectional pairs, which are defined as two genes that lie in a head-to-head arrangement, on opposite DNA strands, with less than $1 \mathrm{~kb}$ separating their transcription start site (TSS).

TFPT, also known as CF3 fusion partner or FB1, encodes a 253 amino acid protein, that was first identified in some cases of paediatric pre-B-cell acute lymphoblastic leukaemia as the fusion partner of the transcription factor E2A [13]. A role for the human protein has not yet been described, but the rat homologue, Tfpt, has been shown to be proapoptotic and might modulate cerebral apoptosis [14].

As differential expression of PRPF31 underlies phenotypic nonpenetrance, a study was undertaken to characterize the core promoter element of the gene (and the bidirectional gene pair, TFPT), as it was considered important to understand the transcriptional regulation of the gene in the normal population [15]. The work by Rose et al. [15] repeated and extended a previous promoter characterization study by Brambillasca et al. [16], which had assayed fragments from the reverse strand in order to characterize a putative TFPT promoter element. Dual luciferase reporter assay was performed and a fragment termed $\mathrm{BiP}$ was defined as the core promoter of $P R P F 31$, whereas the core promoter of TFPT was defined as a fragment termed P.31-Luc [15].

It was considered that studying the conservation and evolution of the PRPF31 and TFPT core promoters in several mammalian species might shed light on the complex regulation of these genes and the failure of mouse models of PRPF31-adRP. The present study design was based on the results of the study on the human genes [15], through the identification of regions homologous to the active human DNA fragments. The homologous genomic regions were tested by dual-luciferase reporter assay in order to assess conservation of gene regulation. Where homology with the human region was found to be low, novel fragments were assayed.

\section{Methods}

\section{Bioinformatic analysis}

Evolutionary conservation of regions was analyzed using ECR browser in NCBI DCODE software suite using default software settings for each program [17].

PAZAR transcription factor work space was used to find TFBS for PRPF31 and TFPT promoters in human and monkey and bidirectional promoters of dog and mouse [18]. Transfac, Jaspar and Oreganno vertebrate profiles were used to define TFBS in conserved promoter regions. For the experimentally-defined mouse promoter region, analysis with classical vertebrate profiles of TF did not identify any TFBS.
In order to find TFBS in mouse, universal protein binding microarray data was used. UNIPROBE database (and its standard TF binding algorithm) was used to find TFBS in experimentally-defined mouse promoter sequence, using strict criterion of enrichment score $=0.49$ [19].

\section{Genomic DNA extraction}

Genomic DNA was isolated from mammalian cell lines using Wizard SV Genomic DNA Purification System (Promega, UK) according to manufacturer's instructions. Monkey DNA was extracted from cos-7 cell line, dog from MDCK cell line and mouse from IMCD3 cell line. All cell lines were purchased from ADCC.

\section{Fragment design and amplification}

The genomic DNA sequence in the three test species was examined and fragments homologous to the three human fragments identified. In the mouse, where homology was limited, we initially looked for any conserved TFBS, but there were none observed. Therefore, fragments surrounding the Prpf31 TSS were designed arbitrarily, according to possibility of PCR amplification in a difficult GC-rich region. In the dog, where the homologous region was approximately 2000bp upstream of Prpf31 TSS, fragments were designed immediately adjacent to the TSS also. The twelve regions of interest were amplified by PCR using KOD polymerase (Novagen) and cloned into pGL3-basic vector (Promega, $\mathrm{UK}$ ) in both forward (indicated by + ) and reverse (indicated by -) strand orientation. Primers and PCR conditions can be seen in Table 6. In total, twelve regions were selected for assay by dual luciferase reporter assay (Figure 2).

\section{Dual luciferase reporter assays}

The pGL3-reporter constructs were transfected into RPE-1 and HeLa cell lines. Additionally, due to concerns about species-specific transcription factor differences, mouse constructs were transfected into IMCD3 cell lines, and dog constructs into MDCK cell lines. Dual luciferase reporter assays were performed in quadruplicate, on three separate occasions. A negative control (pGL3-basic) and positive control (minimal thymidine kinase promoter, pTK) was assayed in each experiment. The transfection protocol and dual-luciferase reporter assay were performed as previously described [15]. Reporter assay data was analysed by firstly standardizing for cell number, by calculating the ratio of firefly luciferase (test) to renilla luciferase (control). This value was then compared to the pTK values, as pTK is considered a goldstandard basic promoter and, therefore, if a fragment has equivalent or greater activity, it can be considered an active regulatory region.

\section{Prpf31 expression studies}

Whole eye and retina tissues were obtained from DBA/2, 129S2/ $\mathrm{Sv}$ and C57Bl/6J adult wild-type mice, ten animals from each strain were used. The whole eye from the right side and the retina from the contralateral side were collected from each animal. Total RNA was extracted using TRIzol kit (Gibco BRL) according to the manufacturer's instructions. cDNA was prepared using the QuantiTect ${ }^{\circ}{ }_{-}$Reverse Transcription kit (Qiagen) and $1 \mu \mathrm{g}$ of RNA as template for each reaction. Real-time PCR was carried out with the GeneAmp 7500 System (Applied Biosystem). The PCR reaction was performed using 1 $\mu \mathrm{l}$ cDNA, $12.5 \mathrm{ml}$ SYBR Green Master Mix (Applied Biosystem) and $400 \mathrm{nM}$ primer. Water was added to make a total reaction volume of $20 \mu \mathrm{l}$. The PCR conditions were as follows: preheating, $50^{\circ} \mathrm{C}$ for 2 min and $95^{\circ} \mathrm{C}$ for $10 \mathrm{~min}$; cycling, 40 cycles of $95^{\circ} \mathrm{C}$ for $15 \mathrm{~s}$ and $60^{\circ} \mathrm{C}$ 


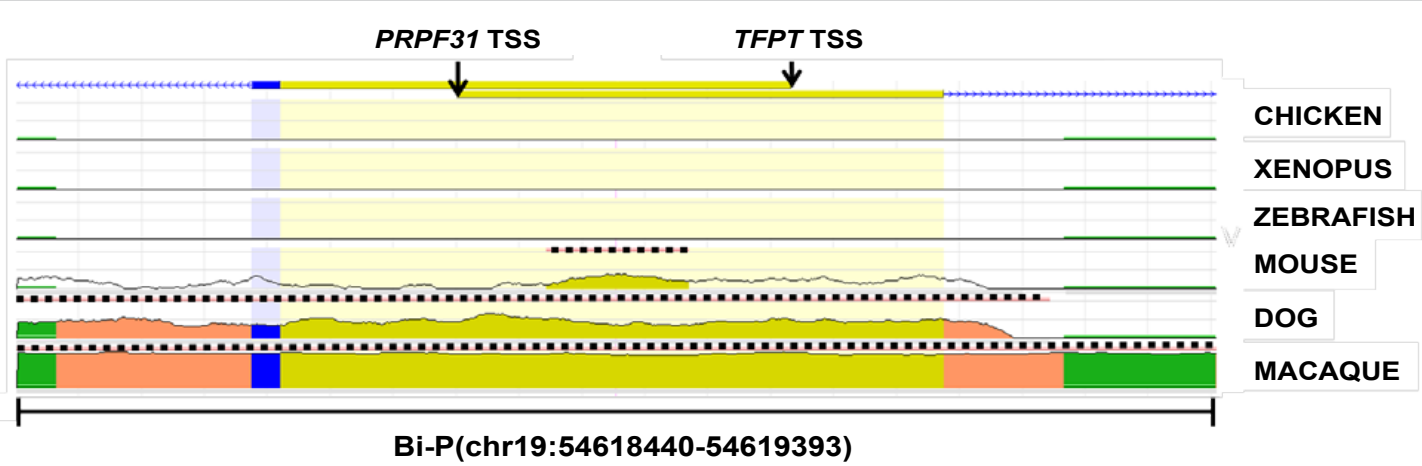

Figure 1: Evolutionary conservation of the Bi-P region, defined as the core promoter for PRPF31 in the human genome. The genome architecture of PRPF31 and TFPT is illustrated, showing exon 1 and TSS of each gene. Degree of conservation is indicated by vertical height of peaks, with areas of significant evolutionary conservation highlighted by a dotted black line. Yellow - non-coding exons, blue - coding exons, salmon pink - introns, green - transposable elements and repeats.

for $1 \mathrm{~min}$. Quantification results were expressed in terms of the cycle threshold $(\mathrm{Ct})$. The $\mathrm{Ct}$ values were averaged for each triplicate. Both the Gapdh (F- GTATGACTCCACTCACGGCAAA; R- TTCCCATTCTCGGCCTTG) and Hprt (F- GAAGAGCTACTGTAATGATCAG; R- GCTGTACTGCTTAACCAGGG) were used as endogenous controls (reference markers). Differences between the mean Ct values of Prpf31 (F- TCGTGTGGACAGCTTCCATG; R- TTCTTCCGCTGCCCATCAAG) and those of the reference genes were calculated as $\Delta \mathrm{Ct}=\mathrm{CT}_{P r p f 31}-\mathrm{CT}_{\text {Hprt (or Gapdh) }}$. Relative fold changes in expression levels were determined as $2^{-\Delta \Delta \mathrm{Ct}}$, the $\operatorname{Prpf} 31$ expression data was normalised with the DBA/2 mouse strain.

\section{Results}

\section{Bioinformatic analysis}

The core promoter of the human PRPF31 gene had previously been identified as Bi-P, the region at chr19:54618440-54619393 (hg19) and the TFPT promoter was contained within this region (chr19:5461844054619133, hg19) [15]. Conservation of the defined regulatory region was analyzed in several species from different lineages, showing a remarkably low level of conservation (Figure 1). It was particularly evident that chicken, Xenopus and zebrafish shared no homology (defined as $<25 \%$ ) with the human region. In the mammalian lineage, macaque and dog shared a high level of homology (defined as $>50 \%$ ) with human, whereas mouse only had a low level homology over a very short distance, the majority of the defined human promoter having no homologous region in mouse. Interestingly, it was noted that although the base sequence was conserved between dog and human, the gene transcription start site (TSS) was different, meaning the homologous sequence in dog was located some 2000bp from the canine Prpf31 TSS.

In light of these findings, three species were selected for study: $C$. sabaeus, $C$. familiaris and M. musculus. It was thought that these species would be interesting, as the green monkey had high homology to the human promoter, the dog had homology in sequence but different genome architecture and the mouse appeared to have different gene regulation entirely.

\section{Definition of core promoter in C. sabaeus}

Three fragments from the C. sabaeus (green monkey) genome that showed very high homology to the human active promoter elements were tested by dual-luciferase reporter assay (Figures 2 and 3, Table 1).

The assay showed that P.31-Luc- had the strongest reporter activity in the reverse strand $($ TFPT) orientation $[2.29 \pm 0.30$ (HeLa); $1.85 \pm$
0.32 (RPE-1)], this confirmed that P.31-Luc was a core promoter with moderate activity, controlling the expression of TFPT in monkey. The TFPT promoter was, therefore, conserved between monkey and human.

In the forward strand (PRPF31) orientation, both Bi-P+ and $\triangle 2+$ had strong promoter activity [Bi-P+: $3.91 \pm 0.52$ (HeLa); $5.12 \pm 0.95$ (RPE-1); $\Delta 2+: 3.74 \pm 0.33$ (HeLa); $5.72 \pm 0.86$ (RPE-1)]. It was clear that both fragments were capable of acting as strong promoter elements. There was no significant difference between the two fragments and, therefore, it was not possible to state unequivocally which was the active core promoter element in vivo. It is likely, however, that Bi-P is the promoter fragment, as $\Delta 2$ does not contain the gene TSS, and would not, therefore, allow correct binding of RNA polymerase II. The $\mathrm{Bi}-\mathrm{P}+$ and $\Delta 2+$ fragments both showed strong promoter activity in human and, as such, the function of these two fragments was conserved between human and green monkey.

\section{Definition of core promoter in C. familiaris}

Initially, two fragments immediately upstream to the $\operatorname{dog} P R P F 31$ TSS were designed and assayed, these fragments being homologous to intron 1 of the human gene (termed $\Sigma 1$ and $\Sigma 2$ ). Luciferase assay in HeLa, RPE-1 and MDCK cell lines showed that these fragments possessed no luciferase activity (Figures 2 and 4A, Table 2).

Subsequently, the fragments that showed homology to the human active promoter fragments - but located 2000bp from Prpf31 TSS were tested by dual luciferase reporter assay in RPE-1, HeLa and MDCK cell lines (Figures 2 and 4B-D, Table 2). This showed that the P.31-Luc fragment was also the core Tfpt promoter in dog, indeed acting as a stronger promoter than that seen in human [6.16 \pm 0.74 (HeLa); 2.40 \pm 0.41 (RPE-1); $4.98 \pm 0.97$ (MDCK)]. The fragment homologous to the human $P R P F 31$ promoter, $\mathrm{Bi}-\mathrm{P}+$, did not have strong promoter activity in dog, with reporter activity less than, or very similar to, pTK $[0.48 \pm 0.09$ (HeLa); $0.91 \pm 0.13$ (RPE-1); $1.16 \pm 0.14$ (MDCK)]. This suggests that the $\mathrm{Bi}-\mathrm{P}+$ fragment does not control the expression of $\operatorname{Prpf} 31$ in the dog.

It was apparent, however, that the constituent elements of $\mathrm{Bi}-\mathrm{P}+$ (P.31-Luc + and $\Delta 2+$ ) had promoter activity, although this was variable between the cell lines tested. In both HeLa and RPE- 1 cell lines, $\Delta 2+$ had the highest promoter activity $[4.71 \pm 0.67(\mathrm{HeLa}) ; 2.58 \pm 0.58$ (RPE-1)], whereas P.31-Luc+ showed only slight activity [1.77 \pm 0.42 (HeLa); $0.99 \pm 0.30$ (RPE-1)]. This situation was not observed in MDCK 


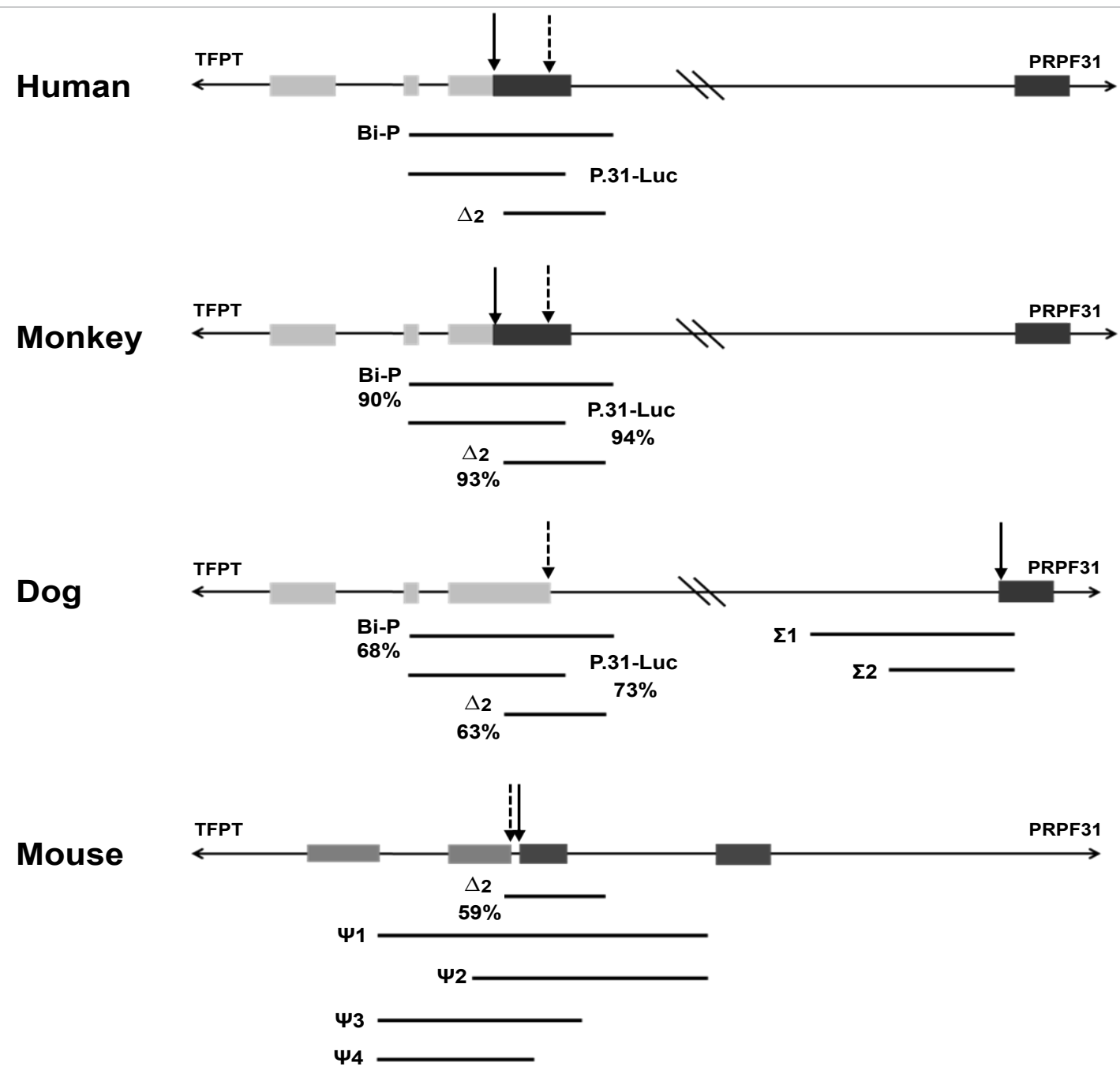

Figure 2: Schematic representation of the genomic regions assayed by dual-luciferase reporter assay. The three fragments with defined reporter activity in human are illustrated, as well as the homologous regions in African green monkey, dog and mouse. The PRPF31 TSS is indicated with a solid arrow, the TFPT TSS with a dashed arrow. Where appropriate, the percentage homology with the human fragment is indicated.

\begin{tabular}{|l|c|c|c|c|}
\hline & HeLa & RPE-1 & HeLa (SD) & RPE-1 (SD) \\
\hline pTK & 1.001 & 1.001 & 0.064 & 0.144 \\
\hline pGL3-empty & 0.008 & 0.082 & 0.004 & 0.018 \\
\hline P.31-Luc - & 2.289 & 1.852 & 0.299 & 0.323 \\
\hline P.31-Luc + & 0.966 & 1.012 & 0.176 & 0.202 \\
\hline$\Delta 2-$ & 1.997 & 0.926 & 0.429 & 0.342 \\
\hline$\Delta 2+$ & 3.744 & 5.716 & 0.326 & 0.864 \\
\hline Bi-P - & 0.322 & 1.168 & 0.089 & 0.432 \\
\hline Bi-P + & 3.907 & 5.118 & 0.522 & 0.947 \\
\hline
\end{tabular}

Table 1: Mean dual luciferase reporter assay ratio values and standard deviation (SD) using sequence from green monkey, assayed in HeLa and RPE-1 cell lines.

cell line, where P.31-Luc+ possessed strong promoter activity [5.01 \pm 0.52 (MDCK)], although $\Delta 2+$ also displayed good reporter activity $[2.65 \pm 0.46(\mathrm{MDCK})]$. This suggested that the strong activation of P.31-Luc+ requires the binding of a dog-species specific transcription factor (TF), and that P.31-Luc is a true bi-directional promoter in $C$. familiaris, controlling the expression of both Prpf31 and Tfpt.

\section{Definition of core promoter in M. musculus}

There was very little homology between the regions surrounding Prpf31 TSS in the mouse genome and the corresponding region in the human genome. Of the three active human fragments (Bi-P, P.31-Luc and $\Delta 2$ ), only $\Delta 2$ had a homologous region in the murine genome (approximately 60\% conservation). Therefore, the $\Delta 2$ fragment and four additional fragments that shared no homology with human regions (termed $\psi 1-4$ ) were assayed by dual-luciferase reporter assay in both forward- and reverse-strand orientations (Figures 2 and 5, Table 3).

In mouse, $\psi 1$ acted as a true bidirectional promoter, controlling the expression of Tfpt and Prpf31. The results were most clear in IMCD3 (murine) cell line, where $\psi 1$ had very strong reporter activity in the forward strand orientation $(6.61 \pm 0.72)$ and the reverse strand orientation $(13.02 \pm 0.75)$. The same effect was observed in HeLa cell line (forward strand: $4.50 \pm 0.60$; reverse strand: $6.25 \pm 0.62$ ). In RPE- 1 cell line, $\psi 1$ had the strongest activity in the forward strand orientation $(2.85 \pm 0.35)$; in the reverse strand orientation the situation was more 
A

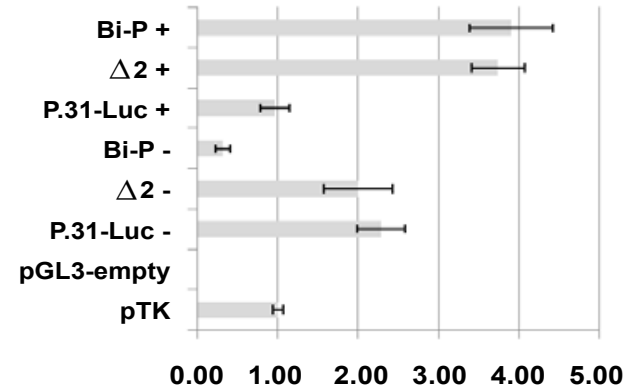

B

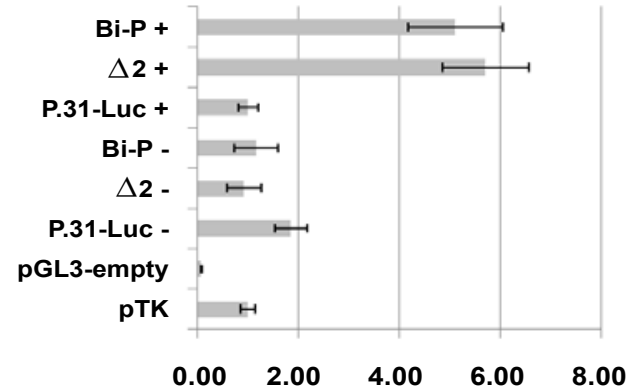

Figure 3: Results of dual-luciferase reporter assay using genomic sequence from C. sabaeus (green monkey) in HeLa cell line (A) and RPE- cell line (B). The data is presented as the average ratio of pGL3-insert to pTK, together with an error bar of \pm one standard deviation, + refers to fragments tested in forward strand (PRPF31) orientation, - to reverse strand (TFPT) orientation. The absolute data values can be seen in Table 1.

\begin{tabular}{|l|c|c|c|c|c|c|}
\hline & HeLa & RPE-1 & MDCK & HeLa (SD) & RPE-1 (SD) & MDCK (SD) \\
\hline pTK & 1.001 & 1.001 & 1.001 & 0.064 & 0.144 & 0.084 \\
\hline pGL3-empty & 0.008 & 0.082 & 0.083 & 0.004 & 0.018 & 0.015 \\
\hline$\Sigma 2-$ & 0.080 & 0.084 & 0.058 & 0.023 & 0.027 & 0.025 \\
\hline$\Sigma 2+$ & 0.062 & 0.058 & 0.043 & 0.025 & 0.015 & 0.012 \\
\hline$\Sigma 1-$ & 0.053 & 0.031 & 0.044 & 0.020 & 0.010 & 0.016 \\
\hline$\Sigma 1+$ & 0.090 & 0.181 & 0.038 & 0.030 & 0.052 & 0.018 \\
\hline P.31-Luc - & 6.164 & 2.399 & 4.979 & 0.743 & 0.414 & 0.967 \\
\hline$\Delta 2-$ & 4.499 & 1.357 & 3.384 & 0.968 & 0.209 & 0.786 \\
\hline Bi-P - & 0.398 & 0.704 & 0.787 & 0.036 & 0.119 & 0.207 \\
\hline P.31-Luc + & 1.766 & 0.995 & 5.011 & 0.419 & 0.299 & 0.528 \\
\hline$\Delta 2+$ & 4.712 & 2.579 & 2.653 & 0.666 & 0.576 & 0.461 \\
\hline Bi-P + & 0.485 & 0.910 & 1.168 & 0.092 & 0.129 & 0.143 \\
\hline
\end{tabular}

Table 2: Mean dual luciferase reporter assay ratio values and standard deviation (SD) using sequence from dog, assayed in HeLa, RPE-1 and MDCK cell lines.

complex, as three fragments had relatively strong reporter activity $[\Delta 2$ : (3.22 \pm 0.26$), \psi 1:(3.76 \pm 0.75), \psi 2:(4.10 \pm 0.22)]$. This result might be due to the different transcription factor profile in RPE-1 cells, and the differences between human and murine transcription factors. Given the clear result in IMCD3 cell line (the most realistic model of the in vivo situation), it was concluded that $\psi 1$ acted as a bidirectional promoter controlling the expression of both Tfpt and Prpf31.

The $\psi 1$ region in mouse shared no significant homology with any human chromosomal region, with only very short regions $(<54 \mathrm{bp})$ of imperfect alignment with human chromosomes 8, 14, 19, 20 and $\mathrm{X}$ (Table 4).

\section{Prediction of transcription factor binding sites}

A bioinformatic approach was taken to identify putative classical TF binding sites (TFBS) within the experimentally-defined promoters in monkey, dog and mouse. The transcription factor workspace of PAZAR was used to look for TFBS in the characterized promoters that showed conservation with human defined promoter. For mouse prediction of TFBS by pairwise conservation between mouse and dog was attempted.

As expected, there was a general overlap of TFBS between human and monkey, for both PRPF31 and TFPT promoters (enriched in signal transduction mechanism and transcription functions) (Figure 6A). Moreover, it was observed that three TFBS (Myf, Gata1 and SP1) were shared between human, monkey and $\operatorname{dog}$ (Figure 6A and 6B). It was not possible, however, to identify any putative classical TFBS in the mouse promoter using pairwise conservation between mouse and human or mouse and dog (scanning for standard vertebrate transcription factor profiles of Jaspar, Transfac \& Oreganno). It was also observed that the human promoter region was enriched with strong $\mathrm{H} 3 \mathrm{~K} 4 \mathrm{Me} 3$ mark for 7 ENCODE cell lines assayed for this histone methylation (Figure 6).

As a conservation based approach could not be used in mouse, due to lack of homology between mouse and human sequence, an analysis was performed using universal protein binding microarray (PBM) data, to identify putative TFBS in the experimentally-defined murine bidirectional promoter $(\psi 1)$. A strict threshold of $0.49 \mathrm{TF}$ enrichment was used and all mouse PBM experiments in UNIPROBE database were searched. A range of TF having strong binding affinity to oligonucleotides of mouse promoter sequence were defined (Figure 7, Table 5). Amongst these, some TF classes were computationally predicted to bind to characterized promoter in monkey and dog (e.g. TCFE2A, zinc finger family, NR2F). However, analysis of functional enrichment showed that TFs binding to the mouse promoters were enriched in purine metabolism and also Hox cluster genes (which are important during development and homeostasis). These findings support the divergent evolution of promoter sequences of human and mouse, by gain of a new function in the mouse lineage.

\section{Study of Prpf31 expression in M. musculus}

In human populations, PRPF31 displays differentially expressed

\begin{tabular}{|l|c|c|c|c|c|c|}
\hline & HeLa & RPE-1 & IMCD3 & HeLa (SD) & RPE-1 (SD) & IMCD3 (SD) \\
\hline pTK & 1.001 & 1.001 & 1.000 & 0.064 & 0.144 & 0.110 \\
\hline pGL3-empty & 0.008 & 0.082 & 0.055 & 0.004 & 0.018 & 0.010 \\
\hline$\Delta 2-$ & 1.910 & 3.219 & 1.822 & 0.258 & 0.447 & 0.192 \\
\hline$\psi 4-$ & 0.403 & 0.523 & 0.396 & 0.147 & 0.134 & 0.098 \\
\hline$\psi 3-$ & 0.659 & 0.318 & 2.059 & 0.185 & 0.042 & 0.202 \\
\hline$\psi 2-$ & 3.296 & 4.095 & 2.283 & 0.222 & 0.570 & 0.276 \\
\hline$\psi 1-$ & 6.253 & 3.758 & 13.023 & 0.624 & 0.753 & 1.356 \\
\hline$\Delta 2+$ & 0.875 & 0.372 & 0.395 & 0.161 & 0.136 & 0.065 \\
\hline$\psi 4+$ & 0.430 & 1.512 & 0.384 & 0.096 & 0.294 & 0.134 \\
\hline$\psi 3+$ & 1.960 & 1.352 & 1.912 & 0.355 & 0.202 & 0.160 \\
\hline$\psi 2+$ & 0.449 & 0.451 & 0.642 & 0.083 & 0.152 & 0.127 \\
\hline$\psi 1+$ & 4.495 & 2.849 & 6.609 & 0.596 & 0.347 & 0.719 \\
\hline
\end{tabular}

Table 3: Mean dual luciferase reporter assay ratio values and standard deviation (SD) using sequence from mouse, assayed in HeLa, RPE-1 and IMCD3 cell lines. 

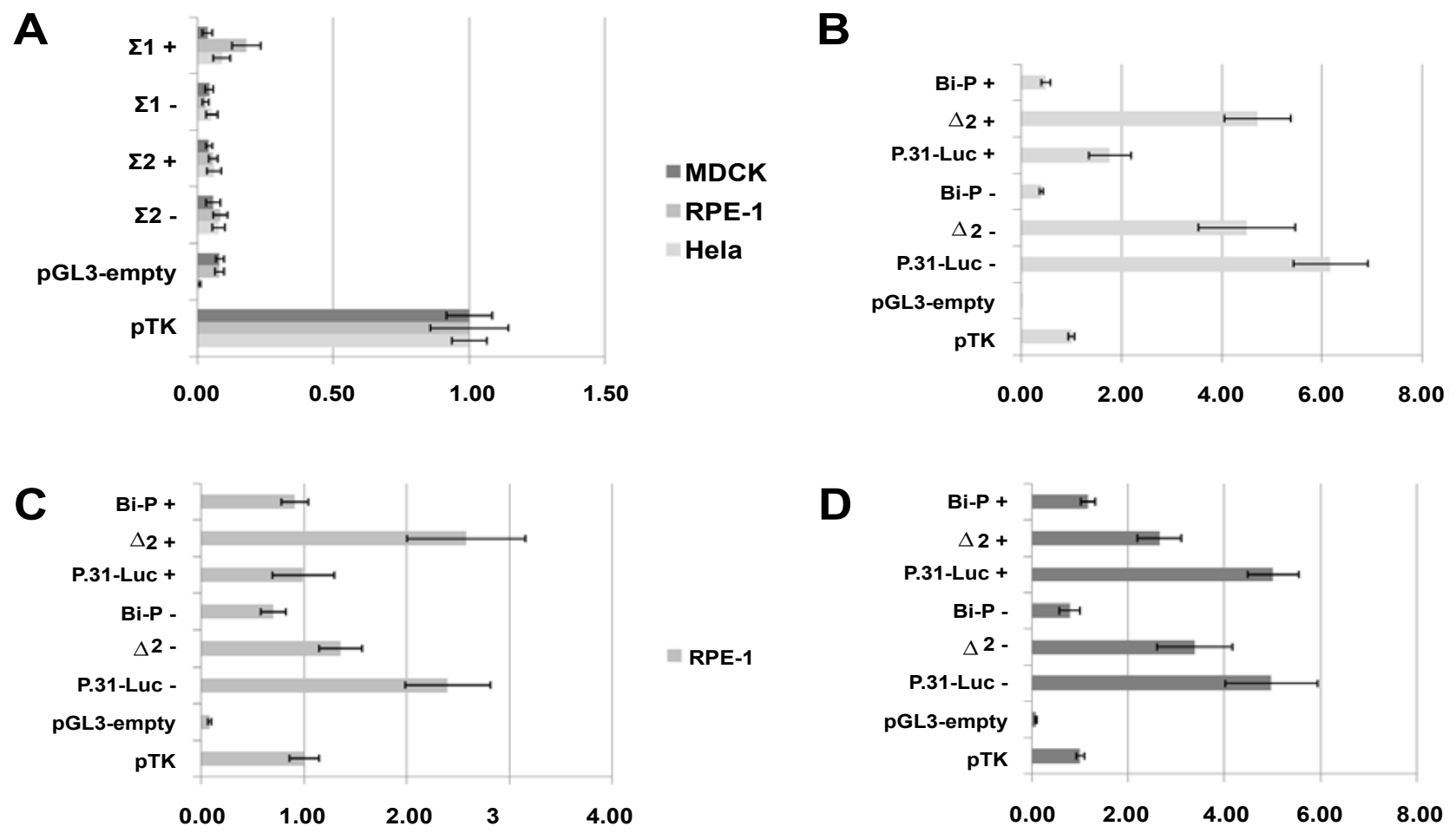

MDCK

Figure 4: Results of dual-luciferase reporter assay using genomic sequence from $C$. familiaris (domestic dog). The region immediately upstream to the gene transcription start site were initially assayed (A), followed by regions located $2000 \mathrm{bp}$ upstream [(B)-Hela, (C) - RPE-1, (D) - MDCK]. The data is presented as the average ratio of pGL3-insert to pTK, together with an error bar of \pm one standard deviation, + refers to fragments tested in forward strand (PRPF31) orientation, - to reverse strand (TFPT) orientation. The absolute data values can be seen in Table 2.
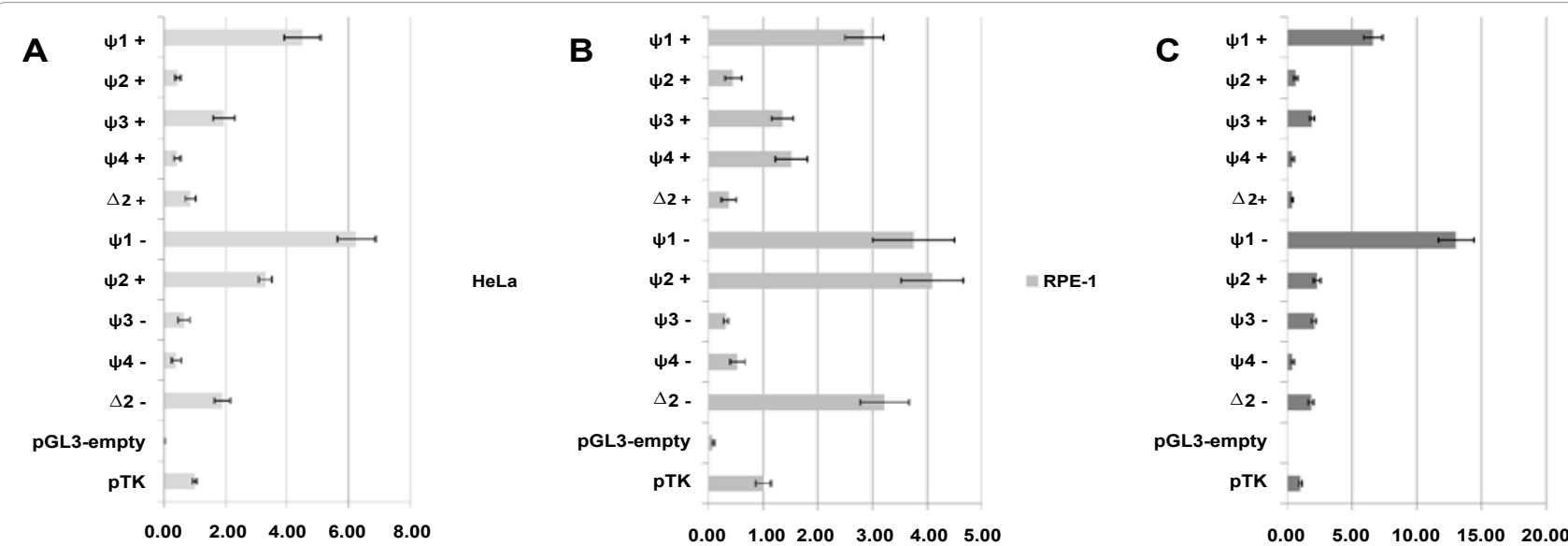

$=\operatorname{IMCD} 3$

Figure 5: Results of dual-luciferase reporter assay using genomic sequence from M. musculus (house mouse) in HeLa cells (A), RPE- cells (B) and IMCD3 cells (C). The data is presented as the average ratio of pGL3-insert to $\mathrm{pTK}$, together with an error bar of \pm one standard deviation, + refers to fragments tested in forward strand (PRPF31) orientation, - to reverse strand (TFPT) orientation. The absolute data values can be seen in Table 3.

\begin{tabular}{|c|c|c|c|c|c|c|}
\hline Accession & Chromosome & Max score & Total score & Query coverage & E value & Max identity \\
\hline NW_003571061.1 & 19 & 42.8 & 42.8 & $4 \%$ & 1 & $78 \%$ \\
\hline NW_003571060.1 & 19 & 42.8 & 42.8 & $4 \%$ & 1 & $78 \%$ \\
\hline NW_003571059.1 & 19 & 42.8 & 42.8 & $4 \%$ & 1 & $78 \%$ \\
\hline NW_003571058.1 & 19 & 42.8 & 42.8 & $4 \%$ & 1 & $78 \%$ \\
\hline NW_003571057.1 & 19 & 42.8 & 42.8 & $4 \%$ & 1 & $78 \%$ \\
\hline NW_003571056.1 & 19 & 42.8 & 42.8 & $4 \%$ & 1 & $78 \%$ \\
\hline
\end{tabular}


Citation: Rose AM, Shah AZ, Alfano G, Bujakowska K, Barker AF, et al. (2013) A Study into the Evolutionary Divergence of the Core Promoter Elements of PRPF31 and TFPT. J Mol Genet Med 7: 67. doi:10.4172/1747-0862.1000067

Page 7 of 12

\begin{tabular}{|c|c|c|c|c|c|c|}
\hline NW_003571055.1 & 19 & 42.8 & 42.8 & $4 \%$ & 1 & $78 \%$ \\
\hline NW_003571054.1 & 19 & 42.8 & 42.8 & $4 \%$ & 1 & $78 \%$ \\
\hline NT_011109.16 & 19 & 42.8 & 42.8 & $4 \%$ & 1 & $78 \%$ \\
\hline NW_001838498.2 & 19 & 42.8 & 42.8 & $4 \%$ & 1 & $78 \%$ \\
\hline NT_011651.17 & $x$ & 41 & 41 & $3 \%$ & 3.5 & $81 \%$ \\
\hline NW_001842380.1 & $x$ & 41 & 41 & $3 \%$ & 3.5 & $81 \%$ \\
\hline NT_011362.10 & 20 & 41 & 41 & $2 \%$ & 3.5 & $84 \%$ \\
\hline NW_001838666.1 & 20 & 41 & 41 & $2 \%$ & 3.5 & $84 \%$ \\
\hline NT_026437.12 & 14 & 46.4 & 46.4 & $2 \%$ & 0.082 & $91 \%$ \\
\hline NW_001838111.1 & 14 & 46.4 & 46.4 & $2 \%$ & 0.082 & $91 \%$ \\
\hline NT_167187.1 & 8 & 41 & 41 & $1 \%$ & 3.5 & $96 \%$ \\
\hline NW_001839128.2 & 8 & 41 & 41 & $1 \%$ & 3.5 & $96 \%$ \\
\hline
\end{tabular}

Table 4: Regions in the human genome that are homologous to the experimentally defined mouse promoter, demonstrating minimal homology.

\begin{tabular}{|c|c|c|c|c|}
\hline Gene Match & K-mer & Reverse Complement & Position & Enrichment Score \\
\hline Zfp410 & ACATCCCA & TGGGATGT & 12 & 0.492169 \\
\hline $\mathrm{Nr} 2 f 2$ & TTGACCCT & AGGGTCAA & 63 & 0.491182 \\
\hline Zfp691 & AGGAGCAC & GTGCTCCT & 90 & 0.494015 \\
\hline Zfp691 & GGAGCACC & GGTGCTCC & 91 & 0.491563 \\
\hline Tcfe2a & ACCACCTG & CAGGTGGT & 228 & 0.492541 \\
\hline Max & CCCACGTG & CACGTGGG & 348 & 0.494126 \\
\hline Bhlhb2 & CCACGTGC & GCACGTGG & 349 & 0.493300 \\
\hline Max & CCACGTGC & GCACGTGG & 349 & 0.497488 \\
\hline Bhlhb2 & CACGTGCC & GGCACGTG & 350 & 0.498400 \\
\hline Max & CACGTGCC & GGCACGTG & 350 & 0.492097 \\
\hline Osr1 & CCAGTAGC & GCTACTGG & 479 & 0.491743 \\
\hline Osr2 & CCAGTAGC & GCTACTGG & 479 & 0.495304 \\
\hline Osr1 & CAGTAGCT & AGCTACTG & 480 & 0.491785 \\
\hline Osr2 & CAGTAGCT & AGCTACTG & 480 & 0.494346 \\
\hline Gabpa & GCTTCCGG & CCGGAAGC & 490 & 0.494322 \\
\hline Gabpa & CTTCCGGC & GCCGGAAG & 491 & 0.493443 \\
\hline Lef1 & TCTTTGAT & ATCAAAGA & 582 & 0.493387 \\
\hline Tcf7I2 & TCTTTGAT & ATCAAAGA & 582 & 0.493092 \\
\hline Tcf7 & CTTTGATG & CATCAAAG & 583 & 0.492072 \\
\hline Tcf3 & CTTTGATG & CATCAAAG & 583 & 0.493912 \\
\hline Lef1 & CTTTGATG & CATCAAAG & 583 & 0.496125 \\
\hline Tcf7l2 & CTTTGATG & CATCAAAG & 583 & 0.496806 \\
\hline Jundm2 & TGATGACG & CGTCATCA & 586 & 0.493965 \\
\hline Jundm2 & GATGACGT & ACGTCATC & 587 & 0.495828 \\
\hline Gm5454 & GCTTCCGG & CCGGAAGC & 490 & 0.495590 \\
\hline Etv1 & GCTTCCGG & CCGGAAGC & 490 & 0.495020 \\
\hline Elk3 & GCTTCCGG & CCGGAAGC & 490 & 0.495800 \\
\hline Etv6 & GCTTCCGG & CCGGAAGC & 490 & 0.492150 \\
\hline Etv4 & GCTTCCGG & CCGGAAGC & 490 & 0.491930 \\
\hline Fli1 & GCTTCCGG & CCGGAAGC & 490 & 0.492160 \\
\hline Elk4 & GCTTCCGG & CCGGAAGC & 490 & 0.491940 \\
\hline Ets1 & GCTTCCGG & CCGGAAGC & 490 & 0.491280 \\
\hline Elf4 & GCTTCCGG & CCGGAAGC & 490 & 0.493140 \\
\hline Etv5 & GCTTCCGG & CCGGAAGC & 490 & 0.495350 \\
\hline Erg & GCTTCCGG & CCGGAAGC & 490 & 0.493290 \\
\hline Elk1 & GCTTCCGG & CCGGAAGC & 490 & 0.494580 \\
\hline Gabpa & GCTTCCGG & CCGGAAGC & 490 & 0.492650 \\
\hline Elf2 & GCTTCCGG & CCGGAAGC & 490 & 0.492000 \\
\hline Gm5454 & CTTCCGGC & GCCGGAAG & 491 & 0.492270 \\
\hline
\end{tabular}


Citation: Rose AM, Shah AZ, Alfano G, Bujakowska K, Barker AF, et al. (2013) A Study into the Evolutionary Divergence of the Core Promoter Elements of PRPF31 and TFPT. J Mol Genet Med 7: 67. doi:10.4172/1747-0862.1000067

Page 8 of 12

\begin{tabular}{|c|c|c|c|c|}
\hline Etv1 & CTTCCGGC & GCCGGAAG & 491 & 0.491880 \\
\hline Elk3 & CTTCCGGC & GCCGGAAG & 491 & 0.494370 \\
\hline Fli1 & CTTCCGGC & GCCGGAAG & 491 & 0.490020 \\
\hline Etv5 & CTTCCGGC & GCCGGAAG & 491 & 0.493190 \\
\hline Elk1 & CTTCCGGC & GCCGGAAG & 491 & 0.491590 \\
\hline Gabpa & CTTCCGGC & GCCGGAAG & 491 & 0.490780 \\
\hline Cphx & TTTGATTG & CAATCAAA & 620 & 0.491680 \\
\hline Lhx8 & TTGATTGG & CCAATCAA & 621 & 0.491150 \\
\hline Cphx & TTGATTGG & CCAATCAA & 621 & 0.496010 \\
\hline Duxl & TTGATTGG & CCAATCAA & 621 & 0.496350 \\
\hline Cphx & TGATTGGC & GCCAATCA & 622 & 0.491310 \\
\hline Hoxd12 & GTTTACGA & TCGTAAAC & 631 & 0.493010 \\
\hline Hoxa13 & GTTTACGA & TCGTAAAC & 631 & 0.492860 \\
\hline Hoxd11 & TTTACGAC & GTCGTAAA & 632 & 0.497210 \\
\hline Hoxc12 & TTTACGAC & GTCGTAAA & 632 & 0.497690 \\
\hline Hoxc13 & TTTACGAC & GTCGTAAA & 632 & 0.490000 \\
\hline Hoxd12 & TTTACGAC & GTCGTAAA & 632 & 0.498500 \\
\hline Hoxb9 & TTTACGAC & GTCGTAAA & 632 & 0.494760 \\
\hline Hoxc11 & TTTACGAC & GTCGTAAA & 632 & 0.498230 \\
\hline Hoxc10 & TTTACGAC & GTCGTAAA & 632 & 0.497220 \\
\hline Hoxa11 & TTTACGAC & GTCGTAAA & 632 & 0.497390 \\
\hline Hoxc13 & TTTATTAG & CTAATAAA & 666 & 0.492680 \\
\hline Hoxa13 & TTTATTAG & CTAATAAA & 666 & 0.493680 \\
\hline Gabpa & ATTTCCGG & CCGGAAAT & 640 & 0.495780 \\
\hline Ehf & ATTTCCGG & CCGGAAAT & 640 & 0.490102 \\
\hline Zbtb12 & GGGTTCTA & TAGAACCC & 707 & 0.492551 \\
\hline Zbtb12 & GGTTCTAG & CTAGAACC & 708 & 0.498015 \\
\hline Zbtb12 & GTTCTAGG & CCTAGAAC & 709 & 0.497873 \\
\hline Gm4881 & CATTTCCG & CGGAAATG & 639 & 0.490280 \\
\hline Erg & CATTTCCG & CGGAAATG & 639 & 0.490560 \\
\hline Gm4881 & ATTTCCGG & CCGGAAAT & 640 & 0.495580 \\
\hline Gm5454 & ATTTCCGG & CCGGAAAT & 640 & 0.495450 \\
\hline Etv3 & ATTTCCGG & CCGGAAAT & 640 & 0.490630 \\
\hline Etv1 & ATTTCCGG & CCGGAAAT & 640 & 0.494530 \\
\hline Elk3 & ATTTCCGG & CCGGAAAT & 640 & 0.493980 \\
\hline Etv6 & ATTTCCGG & CCGGAAAT & 640 & 0.496360 \\
\hline Etv4 & ATTTCCGG & CCGGAAAT & 640 & 0.493710 \\
\hline Fli1 & ATTTCCGG & CCGGAAAT & 640 & 0.497170 \\
\hline Elk4 & ATTTCCGG & CCGGAAAT & 640 & 0.495700 \\
\hline Ets1 & ATTTCCGG & CCGGAAAT & 640 & 0.496500 \\
\hline Elf4 & ATTTCCGG & CCGGAAAT & 640 & 0.495400 \\
\hline Etv5 & ATTTCCGG & CCGGAAAT & 640 & 0.494770 \\
\hline Erg & ATTTCCGG & CCGGAAAT & 640 & 0.496890 \\
\hline Elk1 & ATTTCCGG & CCGGAAAT & 640 & 0.493770 \\
\hline Elf5 & ATTTCCGG & CCGGAAAT & 640 & 0.492260 \\
\hline Gabpa & ATTTCCGG & CCGGAAAT & 640 & 0.496960 \\
\hline Elf3 & ATTTCCGG & CCGGAAAT & 640 & 0.493910 \\
\hline Elf2 & ATTTCCGG & CCGGAAAT & 640 & 0.495170 \\
\hline Ehf & ATTTCCGG & CCGGAAAT & 640 & 0.493370 \\
\hline Zfp691 & TAGTGCTC & GAGCACTA & 1085 & 0.495547 \\
\hline Zfp691 & AGTGCTCT & AGAGCACT & 1086 & 0.493492 \\
\hline
\end{tabular}

Table 5: Transcription factor binding sites identified within the murine promoter, found using mouse PBM experiments in UNIPROBE database. 
Citation: Rose AM, Shah AZ, Alfano G, Bujakowska K, Barker AF, et al. (2013) A Study into the Evolutionary Divergence of the Core Promoter Elements of PRPF31 and TFPT. J Mol Genet Med 7: 67. doi:10.4172/1747-0862.1000067

Page 9 of 12

\begin{tabular}{|l|l|l|l|l|l|}
\hline Species & Fragment & $\mathbf{F}$ & $\mathbf{R}$ & Annealing Temp & Extension Time \\
\hline Monkey & $\Delta 2$ & GGAGAATCGTTTGAACCCTGGAGAC & CCACAGCAATTCTCCGCTTAGCAG & 63 & 10,30 \\
\hline & P.31-Luc & GATGACGTCTCATGCTCGCGCC & GCAGTCCAAACCCCTAGCCT & 63 & 14,43 \\
\hline & Bi-P & GGAGAATCGTTTGAACCCTGGAGAC & GCAGTCCAAACCCCTAGCCT & 63 & 20,60 \\
\hline Dog & $\Sigma 1$ & CTCTACAGCCCACTCACCATCTTG & GCTCTTTTCCACATCATGGGAC & 63 & 27,82 \\
\hline & $\Sigma 2$ & CTCTACAGCCCACTCACCATCTTG & CCTAGTTCTGGGAGGTTTGTTC & 63 & 14,43 \\
\hline & $\Delta 2$ & GCTCTAGCGACCTAAACCCGTCTC & CTAGGGCGATGCTCCGCTTAGCA & 63 & 11,33 \\
\hline & P.31-LuC & CCCACTGATGACGTCCCAGGC & GAACTCCAACTCCTAGCTTTTTTCC & 63 & 14,43 \\
\hline & Bi-P & GCTCTAGCGACCTAAACCCGTCTC & GAACTCCAACTCCTAGCTTTTTTCC & 63 & 20,60 \\
\hline & $\Delta 2$ & CTTTGCCTAACCCGGCTCTT & CCAAAGCGAATCTCTGTTTAGC & 60 & 10,30 \\
\hline & $\Psi 1$ & CATCAGCCAGAGACATCCCAAG & CAGACCCTAGCCTCCTCCCTC & 60 & 25,75 \\
\hline & $\Psi 2$ & CATCAGCCAGAGACATCCCAAG & CCAAAGCGAATCTCTGTTTAGC & 57 & 16,48 \\
\hline & $\Psi 3$ & CTTTGCCTAACCCGGCTCTT & CAGACCCTAGCCTCCTCCCTC & 57 & 20,60 \\
\hline & $\Psi 4$ & GGCTCTCTTTGATGACGTTTAC & CAGACCCTAGCCTCCTCCCTC & 57 & 14,40 \\
\hline
\end{tabular}

Table 6: Primer sequence and PCR conditions used for production of genomic fragments.

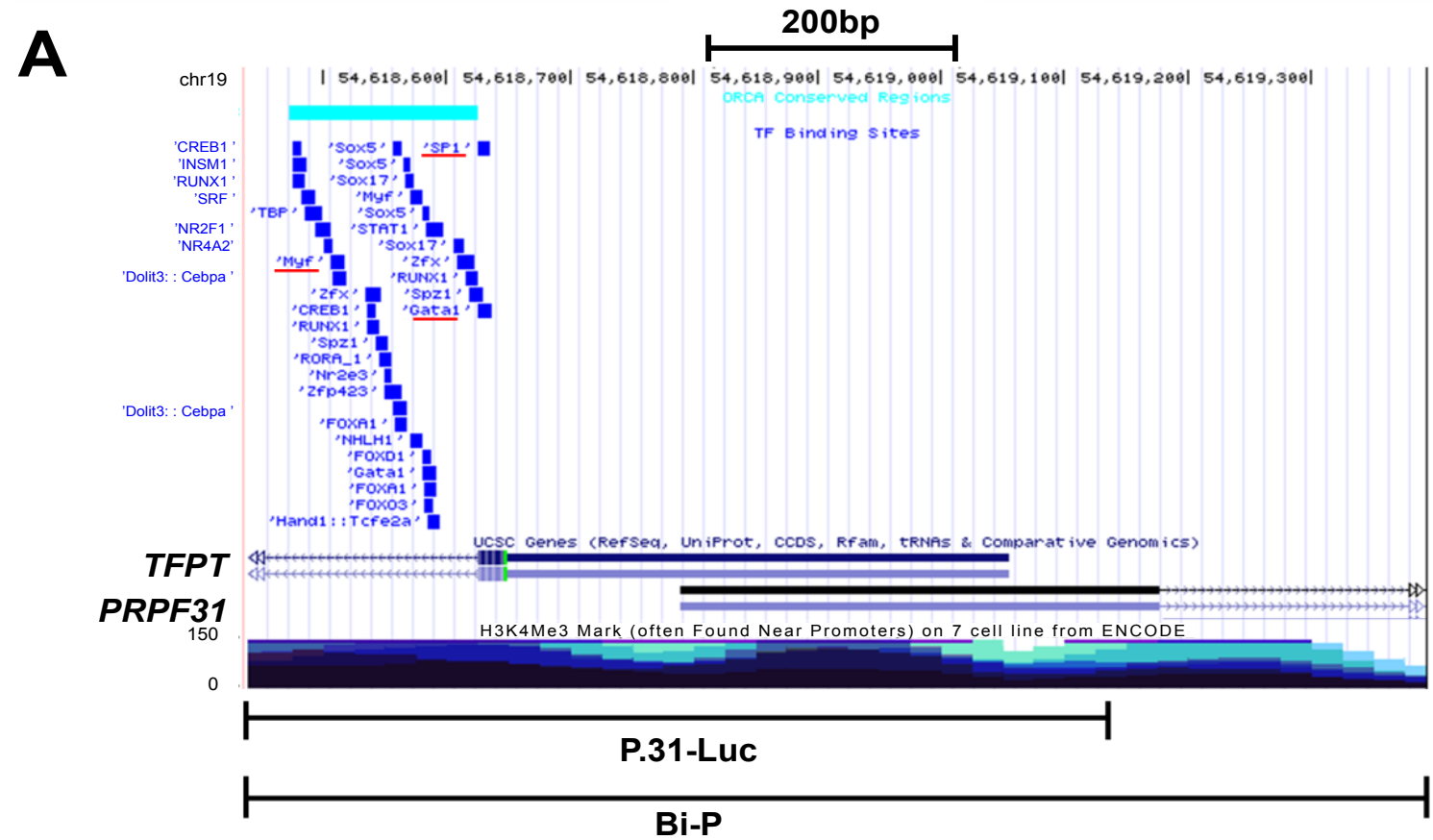

ORCA regions

TFBS

Genes

H3K4Me3 marks

Promoter

fragments

B

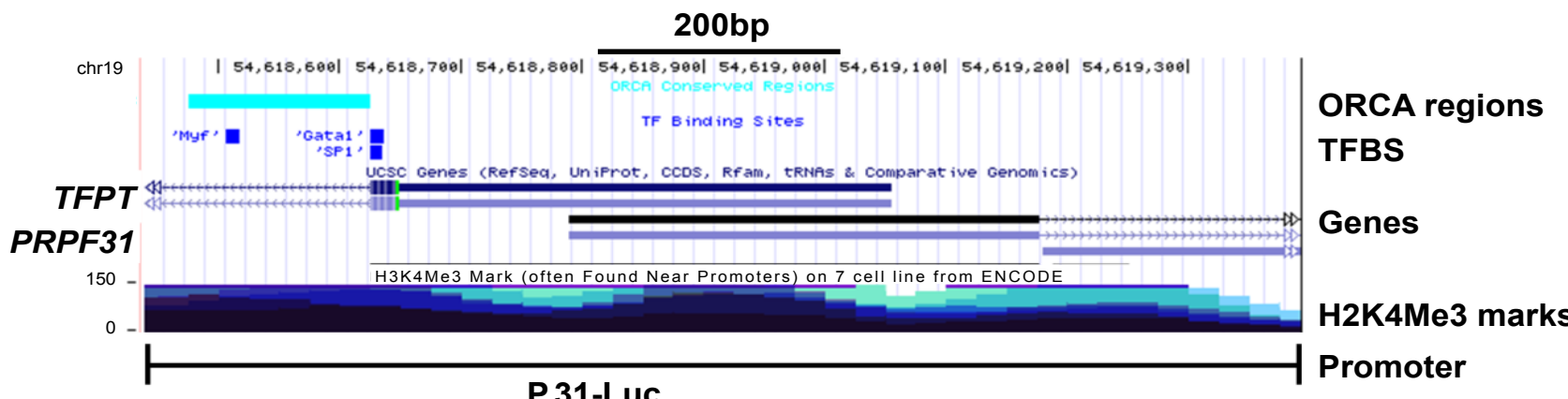

Figure 6: Evolutionary conserved transcription factor binding sites (TFBS) in the experimentally-defined promoters of PRPF31 and TFPT in monkey (A) and dog (B). TFBS conserved between all three species (human, monkey and dog) are underlined in red on (A). ORCA conserved regions highlight areas with high level of evolutionary conservation between human and the test species. The ENCODE derived H3K4Me3 marks indicate areas that are often found in, or near, promoters. 
wild-type alleles, with highly expressed alleles providing protection against the clinical manifestation of PRPF31-associated adRP. We sought to analyze whether there was variable expression of $\operatorname{Prpf} 31$ in M. musculus. Real time qPCR experiments were performed, to quantify Prpf31 expression levels in mouse eyes and retinas. In order to avoid interference due to the genetic background or the age, animals belonging to three different wildtype mouse strains (DBA/2, 129S2/Sv and $\mathrm{C} 57 \mathrm{Bl} / 6 \mathrm{~J}$ ) and of the same age (8 weeks old) were analyzed. To reach a statistical significant number of tested individuals, thirty mice (ten mice for each strain) were analyzed. Prpf31 expression level was tested in the eye and the contralateral retina of each mouse. Experiments were performed using Hprt and Gapdh genes as endogenous control. There was no statistically significant difference in $\operatorname{Prpf} 31$ expression levels between the three mouse strains, either comparing eye or retinal cDNAs (Figure 8). Overall, our data suggest that in the mouse population there is no differential expression of Prpf31 alleles.

\section{Discussion}

The aim of this investigation was to identify and characterize the core promoters controlling the expression of PRPF31 and TFPT in three species, green monkey (C. sabaeus), domestic dog (C. familiaris) and house mouse (M. musculus).

In green monkey, the core promoter of TFPT was defined as a fragment (P.31-Luc) spanning -354 to +355 relative to the TFPT TSS, with comparatively weak promoter activity. It was more difficult to define the PRPF31 promoter in monkey, as both Bi-P+ and $\Delta 2+$ had strong, and very similar, promoter activity. It was, however, considered unlikely that $\Delta 2$ fragment is the true core promoter element of PRPF31, as it does not flank the TSS. It is apparent, however, that this fragment is capable of acting as a promoter in vitro and is likely, therefore, to harbour a RNA polymerase II binding site and other TFBS. As Bi-P spans the PRPF31 TSS $(-406$ to +584$)$, it is more likely that Bi-P is the in vivo promoter, as this would allow correct binding of the RNA polymerase II. The two defined core promoters, P.31-Luc and Bi-P, are homologous to the experimentally-defined human promoters [15]. Furthermore, there were a large number of evolutionarily conserved TFBS between human and monkey species. This was to be expected, as the green monkey fragments share $>90 \%$ homology and there has, therefore, been conservation of the active promoter elements.

In dog, P.31-Luc was defined as a true bidirectional promoter, controlling the expression of both Prpf31 and Tfpt. P.31-Luc spanned $-510 \mathrm{bp}$ to $+208 \mathrm{bp}$ relative to the Tfpt TSS in the dog genome, and had strong promoter activity in this orientation in the three tested cell lines. The fragment shared $73 \%$ homology with the human TFPT promoter, so both the sequence and the function of this region were conserved between the domestic dog and humans.

The region immediately upstream to canine Prpf31 TSS had no reporter activity; but, rather, a long-range promoter was shown to control the expression of Prpf31 in dog. P.31-Luc was defined as the canine core promoter, and spanned -2580 to -1857 relative to the Prpf31 TSS (genomic co-ordinates chr1:103068699-103069421, canFam3). Long-range promoter elements bind RNA polymerase II at the TSS (in the same manner as canonical promoters), but distally bound TFs are later brought into close apposition to the gene TSS by DNA looping, this allowing activation of the RNA polymerase II complex. It is unclear

\section{Mouse chr7:3629316-3630581}

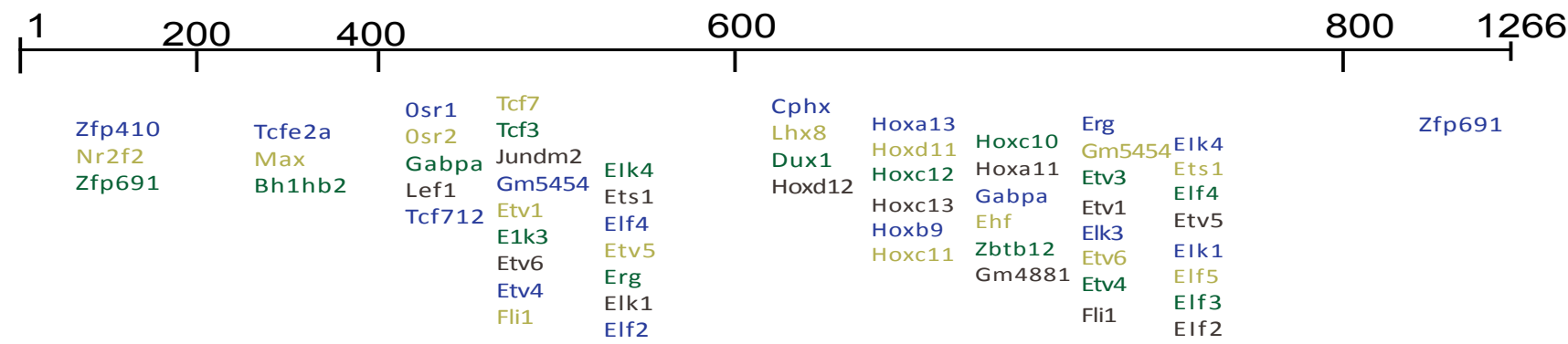

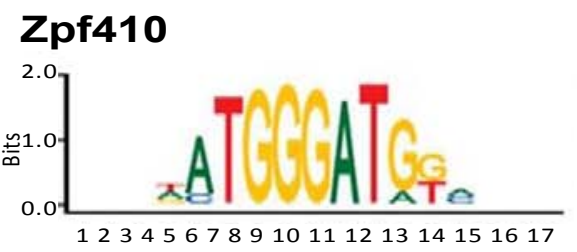

Tcfe2a

Ehf

\section{Zpf691}
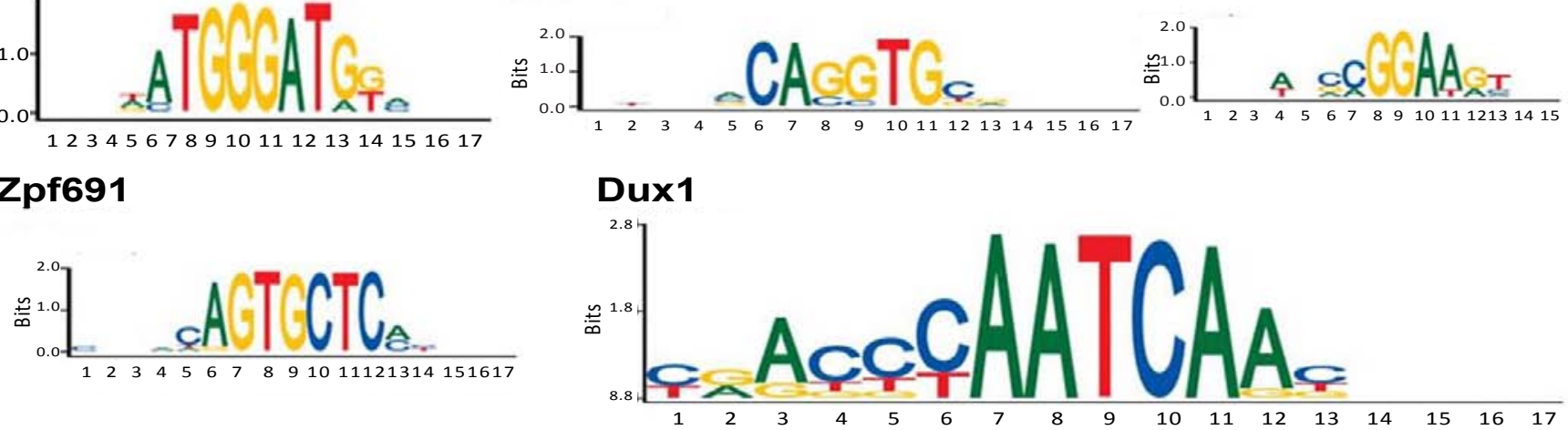

Figure 7: Transcription factor binding sites in the experimentally defined mouse bidirectional promoter ( $\psi 1$, located at murine chromosome 7:3629316-3630581, mm10), that were derived using protein binding microarray data, and oligonucleotide motifs of some representative transcription factors. 


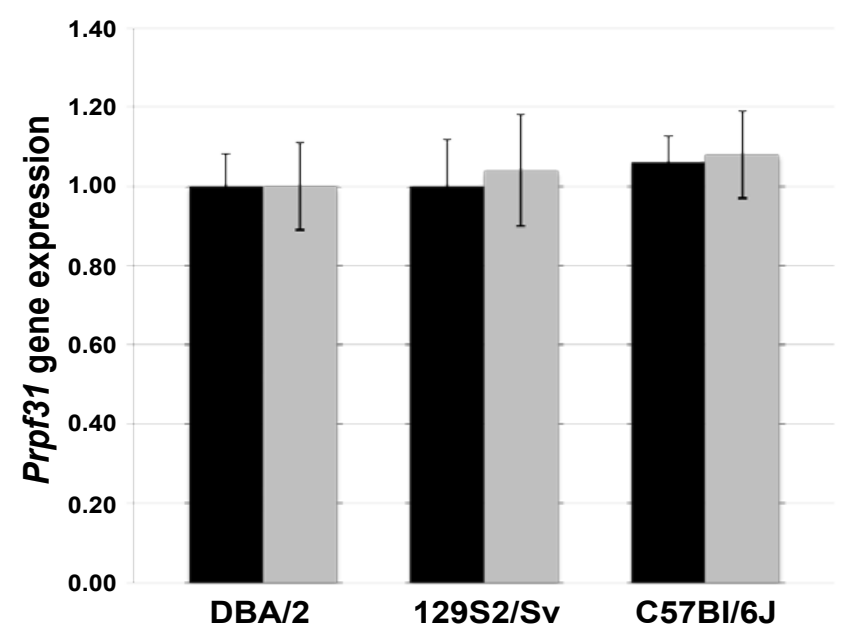

Figure 8: Expression of Prpf31 in whole eye (black bars) and retina (grey bars) of three mouse strains. Data are normalized with the DBA/2 mouse strain. Error bars represent one standard error.

why the sequence that is homologous to the primate exon 1 is not transcribed in the canine lineage.

Interestingly, the Bi-P fragment, which shared $68 \%$ homology with the human PRPF31 core promoter did not show reporter activity. The difference between humans and dog indicates that the functional TFBS in Bi-P have been lost in dog, or that new functional sites have evolved since the divergence of the primate and canine lineages; the latter is more likely, as functional domains tend to have a positive selection pressure and are, therefore, rarely lost through evolution. Analysis of TFBS identified three binding sites that were conserved between human, monkey and dog, all of which are located within the human/ canine P.31-Luc portion, which correlates with the experimental findings.

Furthermore, it should be noted that the human CNOT3 binding site was not conserved in either monkey or dog, despite the higher level of homology between the three species. It remains to be seen whether this is reflected in less variable PRPF31 expression levels in wild populations of these species, given that variable CNOT3 expression was described as an important modulator of PRPF31 expression in the human genome [19]. Another interesting finding of the TFBS prediction was the finding of a conserved NR2E3 binding site between human and monkey. NR2E3 is a transcription factor that plays an important role in developmental differentiation of the photoreceptors and, after development, is specifically expressed in post-mitotic photoreceptors [20]. As such, regulation of PRPF31 by this factor might be part of the explanation of the retina-specific phenotype of PRPF31 mutations.

A mouse fragment, $\psi 1$ (chr7:3629316-3630581, mm10), was defined as a bidirectional promoter controlling the expression of both Tfpt and Prpf31. It was notable that the murine Prpf31 5' region shared little homology with the human $\operatorname{Prpf} 315^{\prime}$ region; indeed, the region that controlled expression of Prpf 31 in mouse has no homologous region in man. Bioinformatic analysis of conserved TFBS demonstrated that the mouse promoter was not regulated by any shared putative TFs with either human or dog. Instead, the mouse bidirectional promoter was enriched with TFBS for Hox family TFs and purine metabolism TFs, indicating significant divergent evolution in the mouse lineage.
It is necessary to speculate on the bidirectional gene architecture of the PRPF31-TFPT gene pair, which is conserved throughout the mammalian lineage, as well as in other vertebrate species, such as chicken, Xenopus, anole lizard, Pelodiscus turtle and even the coelacanth. The phenomenon of bidirectional gene pairs has been observed across most genomes, including yeast, nematode, fish and mammalian and it has been estimated that up to $10 \%$ of human genes exist in this divergent arrangement [21]. Bidirectional genes are controlled by a bidirectional promoter and this might allow cotranscription of the two genes, in a way similar to the prokaryotic operon. Bidirectional promoters are characterized by $\mathrm{CpG}$ islands, that overlap the exon 1 of both genes and this strongly suggests that the level of gene expression in bidirectional gene pairs is controlled by $\mathrm{CpG}$ methylation $[21,22]$. It is unclear why the bidirectional TFPT-PRPF31 gene pair has arisen, given that the two genes share neither protein function nor temporal expression. PRPF31 is a ubiquitously expressed spliceosome component, whilst TFPT is involved in p53-independent cellular apoptosis and is thus mainly active under conditions of cellular stress. It is difficult to imagine a shared selection pressure that might have influenced the gene architecture of these two very different genes. It should be noted, however, that during cellular stress the splicing machinery genes are down-regulated and, as such, the bidirectional gene architecture might bear relevance to the complex changes in gene expression that occur in situations of cellular stress [23].

In this work, it was also demonstrated that there is not variable expression of $\operatorname{Prpf} 31$ in a population of mice from three different strains. This suggests that the differential expression of Prpf 31 has arisen after the evolutionary divergence of rodent and primate lineages. It could be inferred that the different 5' architecture of the two genes is responsible for the lack of differential expression in the mouse. It has been demonstrated that one major factor that determines human $P R P F 31$ expression level is repression of transcription by binding of CNOT3 to the PRPF31 core promoter [9]. As the CNOT3 binding site is not conserved between mouse and human, it follows that differential gene expression is not observed in mouse populations. The different gene regulation is a plausible explanation of why mouse models of human Prpf31 mutations have failed to yield a disease phenotype $[11,12]$. Furthermore, other cis-acting factors within the PRPF31 5 region that are present in human, but not in mouse, might contribute to the observed phenotypic differences.

It appears then, that in evolutionary terms, the high-expressing allele is older and, since the divergence of rodents and primate lineages, a lower-expressing allele has evolved. This raises complex evolutionary questions that will need to be addressed through systematic bioinformatic analysis of phylogenetic and genomic sequencing data. These analyses might lead to a deeper understanding of this unusual situation, whereby there appears to be rapidly-evolving control of an evolutionarily-conserved gene, with different regulatory mechanisms in relatively closely related species.

\section{Acknowledgements}

The authors are grateful for research support from the Rosetrees Trust Butterfield Trust (Bermuda), Foundation Fighting Blindness (USA), Fight fo Sight, RP Fighting Blindness and the Special Trustees of Moorfields Eye Hospital London, UK.

\section{Web Resources}

ECR BROWSER http://ecrbrowser.dcode.org/

PAZAR http://www.pazar.info/

UNIPROBE 
Citation: Rose AM, Shah AZ, Alfano G, Bujakowska K, Barker AF, et al. (2013) A Study into the Evolutionary Divergence of the Core Promoter Elements of PRPF31 and TFPT. J Mol Genet Med 7: 67. doi:10.4172/1747-0862.1000067

\section{References}

1. Vithana EN, Abu-Safieh L, Allen MJ, Carey A, Papaioannou M, et al. (2001) A human homolog of yeast pre-mRNA splicing gene, PRP31, underlies autosomal dominant retinitis pigmentosa on chromosome 19q13.4 (RP11). Mol Cell 8: 375-381.

2. Waseem NH, Vaclavik V, Webster A, Jenkins SA, Bird AC, et al. (2007) Mutations in the gene coding for the pre-mRNA splicing factor, PRPF31, in patients with autosomal dominant retinitis pigmentosa. Invest Ophthalmol Vis Sci 48 : $1330-1334$

3. Rio Frio T, Civic N, Ransijn A, Beckmann JS, Rivolta C (2008) Two trans-acting eQTLs modulate the penetrance of PRPF31 mutations. Hum Mol Genet 17 3154-3165.

4. Rivolta C, McGee TL, Rio Frio T, Jensen RV, Berson EL, et al. (2006) Variation in retinitis pigmentosa-11 (PRPF31 or RP11) gene expression between symptomatic and asymptomatic patients with dominant RP11 mutations. Hum Mutat 27: 644-653.

5. Vithana EN, Abu-Safieh L, Pelosini L, Winchester E, Hornan D, et al. (2003) Expression of PRPF31 mRNA in patients with autosomal dominant retinitis pigmentosa: a molecular clue for incomplete penetrance? Invest Ophthalmo Vis Sci 44: 4204-4209.

6. Liu JY, Dai X, Sheng J, Cui X, Wang X, et al. (2008) Identification and functiona characterization of a novel splicing mutation in RP gene PRPF31. Biochem Biophys Res Commun 367: 420-426.

7. McGee TL, Devoto M, Ott J, Berson EL, Dryja TP (1997) Evidence that the penetrance of mutations at the RP11 locus causing dominant retinitis pigmentosa is influenced by a gene linked to the homologous RP11 allele. Am J Hum Genet 61: 1059-1066.

8. Rio Frio T, Wade NM, Ransijn A, Berson EL, Beckmann JS, et al. (2008) Premature termination codons in PRPF31 cause retinitis pigmentosa via haploinsufficiency due to nonsense-mediated mRNA decay. J Clin Invest 118: 1519-1531.

9. Venturini G, Rose AM, Shah AZ, Bhattacharya SS, Rivolta C (2012) CNOT3 is a modifier of PRPF31 mutations in retinitis pigmentosa with incomplete penetrance. PLoS Genet 8: e1003040.

10. Miller JE, Reese JC (2012) Ccr4-Not complex: the control freak of eukaryotic cells. Crit Rev Biochem Mol Biol 47: 315-333.

11. Bujakowska K, Maubaret C, Chakarova CF, Tanimoto N, Beck SC, et al. (2009) Study of gene-targeted mouse models of splicing factor gene Prpf31 implicated in human autosomal dominant retinitis pigmentosa (RP). Invest Ophthalmol Vis Sci 50: 5927-5933

12. Graziotto JJ, Farkas MH, Bujakowska K, Deramaudt BM, Zhang Q, et al. (2011) Three gene-targeted mouse models of RNA splicing factor RP show late-onset RPE and retinal degeneration. Invest Ophthalmol Vis Sci 52: 190-198.

13. Brambillasca F, Mosna G, Colombo M, Rivolta A, Caslini C, et al. (1999) Identification of a novel molecular partner of the E2A gene in childhood leukemia. Leukemia 13: 369-375.

14. Irie Y, Yamagata K, Gan Y, Miyamoto K, Do E, et al. (2000) Molecular cloning and characterization of Amida, a novel protein which interacts with a neuronspecific immediate early gene product arc, contains novel nuclear localization signals, and causes cell death in cultured cells. J Biol Chem 275: 2647-2653.

15. Rose AM, Shah AZ, Waseem NH, Chakarova CF, Alfano G, et al. (2012) Expression of PRPF31 and TFPT: regulation in health and retinal disease. Hum Mol Genet 21: 4126-4137.

16. Brambillasca F, Mosna G, Ballabio E, Biondi A, Boulukos KE, et al. (2001) Promoter analysis of TFPT (FB1), a molecular partner of TCF3 (E2A) in childhood acute lymphoblastic leukemia. Biochem Biophys Res Commun 288 1250-1257.

17. Ovcharenko I, Nobrega MA, Loots GG, Stubbs L (2004) ECR Browser: a tool for visualizing and accessing data from comparisons of multiple vertebrate genomes. Nucleic Acids Res 32: W280-286.

18. Portales-Casamar E, Arenillas D, Lim J, Swanson MI, Jiang S, et al. (2009) The PAZAR database of gene regulatory information coupled to the ORCA toolkit for the study of regulatory sequences. Nucleic Acids Res 37: D54-60.

19. Robasky K, Bulyk ML (2011) UniPROBE, update 2011: expanded content and search tools in the online database of protein-binding microarray data on protein-DNA interactions. Nucleic Acids Res 39: D124-128.

20. Swaroop A, Kim D, Forrest D (2010) Transcriptional regulation of photoreceptor development and homeostasis in the mammalian retina. Nat Rev Neurosci 11 563-576.

21. Trinklein ND, Aldred SF, Hartman SJ, Schroeder DI, Otillar RP, et al. (2004) An abundance of bidirectional promoters in the human genome. Genome Res 14: $62-66$

22. Adachi N, Lieber MR (2002) Bidirectional gene organization: a common architectural feature of the human genome. Cell 109: 807-809.

23. Biamonti G, Caceres JF (2009) Cellular stress and RNA splicing. Trends Biochem Sci 34: 146-153. 